\title{
Improving What Cross-Modal Retrieval Models Learn through Object-Oriented Inter- and Intra-Modal Attention Networks
}

\author{
Po-Yao Huang \\ Carnegie Mellon University \\ Pittsburgh, PA \\ poyaoh@cs.cmu.edu \\ Xiaojun Chang \\ Monash University \\ Melbourne, VIC \\ cxj273@gmail.com
}

\author{
Vaibhav \\ Carnegie Mellon University \\ Pittsburgh, PA \\ vvaibhav@andrew.cmu.edu \\ Alexander G. Hauptmann \\ Carnegie Mellon University \\ Pittsburgh, PA \\ alex@cs.cmu.edu
}

\begin{abstract}
Although significant progress has been made for cross-modal retrieval models in recent years, few have explored what those models truly learn and what makes one model superior to another. Start by training two state-of-the-art text-to-image retrieval models with adversarial text inputs, we investigate and quantify the importance of syntactic structure and lexical information in learning the joint visual-semantic embedding space for cross-modal retrieval. The results show that the retrieval power mainly comes from localizing and connecting the visual objects and their cross-modal counterparts, the textual phrases. Inspired by this observation, we propose a novel model which employs object-oriented encoders along with inter- and intra-modal attention networks to improve inter-modal dependencies for cross-modal retrieval. In addition, we develop a new multimodal structure-preserving objective which additionally emphasizes intra-modal hard negative examples to promote intra-modal discrepancies. Extensive experiments show that the proposed approach outperforms the existing best method by a large margin (16.4\% and 6.7\% relatively with Recall@1 in the text-toimage retrieval task on the Flickr30K dataset and the MS-COCO dataset respectively).
\end{abstract}

\section{KEYWORDS}

Cross Modal Retrieval, Text-Image Matching, Joint Embedding

\section{ACM Reference Format:}

Po-Yao Huang, Vaibhav, Xiaojun Chang, and Alexander G. Hauptmann. 2019. Improving What Cross-Modal Retrieval Models Learn through ObjectOriented Inter- and Intra-Modal Attention Networks. In International Conference on Multimedia Retrieval (ICMR '19), June 10-13, 2019, Ottawa, ON, Canada. ACM, New York, NY, USA, 9 pages. https://doi.org/10.1145/3323873. 3325043

Permission to make digital or hard copies of all or part of this work for personal or classroom use is granted without fee provided that copies are not made or distributed for profit or commercial advantage and that copies bear this notice and the full citation on the first page. Copyrights for components of this work owned by others than ACM must be honored. Abstracting with credit is permitted. To copy otherwise, or republish, to post on servers or to redistribute to lists, requires prior specific permission and/or a fee. Request permissions from permissions@acm.org.

ICMR '19, June 10-13, 2019, Ottawa, ON, Canada

(c) 2019 Association for Computing Machinery.

ACM ISBN 978-1-4503-6765-3/19/06...\$15.00

https://doi.org/10.1145/3323873.3325043

\section{INTRODUCTION}

Compared to information retrieval within the same modality, matching and retrieving data across modalities remains a long-standing challenge in multimedia retrieval. In text-to-image retrieval task (i.e., searching images with natural language queries), a system needs to exploit both fine-grained intra-modal discrepancies and inter-modal dependencies. For instance, the system has to distinguish the phrase "river bank" from "financial bank" (intra-modal discrepancies) and connect them to the visual objects accordingly (inter-modal dependencies).

To disentangle relationships shared among multimodal data, most cross-modal retrieval models learn a joint embedding space [6, $7,18,23,26,30,39,40]$ in which distances between visual and textual instances are measurable and comparable. In the past few years, a rich line of research including advanced encoders [26, 44], attention mechanisms $[15,30]$, and structure-preserving loss functions [23, 39, 40] or multi-tasking [13] promoted sophisticated approaches to learn improved visual-semantic embeddings. However, few have systematically explored how those models learn the joint embedding space and what aspects of data and model are critical.

To gain insights answering these questions, we perform a series of analyses inspired by $[4,16]$ to train cross-modal retrieval models with perturbed inputs. First, we investigate how well does current state-of-the-art image-to-text retrieval models $[6,26]$ incorporate linguistic structure with recurrent neural networks $[3,11]$ (RNNs). We perform random shuffling on the input sentences during the training phase and measure the performance drop. Surprisingly, we observe that those models are actually insensitive to random shuffling of the word order, indicating that the linguistic structure has very limited impact and is likely to be negligible for crossmodal retrieval. The intuitive follow-up question is: If syntactic structure is not important, what do the models learn? To answer this question, we design experiments to eliminate one lexical type from the textual inputs at a time and measure the degradation. We observe that those models mainly learn to correlate adjectives and nouns to the visual inputs. In contrast, word tokens corresponding to verbs, propositions and counts are almost irrelevant. This result suggests that text-to-image alignment in the joint embedding space is (noun) phrase-oriented, or visually speaking, object-oriented.

Inspired by this observation, we propose a novel object-oriented attention network (OAN) for cross-modal retrieval. First, to capture 
the phrase-level links, we consider convolutional neural networks (CNNs) as the feasible architecture to encode sentences since they naturally capture phrase-level information [20]. To improve the object-oriented links both regionally and globally across modalities, we propose to jointly leverage inter-modal attention and intramodal attention. Furthermore, as mainly noun phrases contribute to the model's performance, this dominance aggravates the problem of many-to-many nature of correspondence in text-image retrieval tasks. To combat this problem, we propose to learn to preserve the neighborhood structure in individual modalities with a new loss function. Different from [6, 40], we emphasize both the inter-modal and the intra-modal hard negative examples.

Our contributions are summarized as follows:

- We present a novel empirical analysis of current state-of-theart cross-modal retrieval systems, showing their strength and limitation then revealing insights for improvements.

- Inspired by this analysis, we propose a novel model with object-oriented encoders, inter- and intra-modal attention networks, and a new multimodal structure-preserving objective to associate object-level embeddings regionally and globally for cross-modal retrieval.

- The proposed model exhibits new state-of-the-art results on the widely-used Flickr30K and MS-COCO datasets.

\section{RELATED WORKS}

Much research has been devoted to learning cross-view representations for retrieving instance across modalities. Broadly, there are two types of representation studied: coordinated embeddings and joint embeddings. In $[12,13,23,41]$, canonical correlation analysis (CCA) is used to capture the association of images and text with coordinated representations. The cons of CCA-based methods are the instability and the computation overhead for calculating the covariance matrix as reported in [40]. Recently, there has been significant interest in learning a joint visual-semantic space $[6,7,18,26,30,39,40]$ for cross-modal retrieval. These models vary mainly in the modality-dependent encoders and the loss functions for learning the joint embedding space.

For encoding text, word embeddings $[29,32]$ and its Fisher vectors [33] have been utilized in [7, 23, 39, 40]. RNNs [3,11] have been used by most cross-modal retrieval models [6, 14, 15, 17-19, 26, 30] while convolution structures have been suggested by [44]. For encoding images, VGG [35] and ResNet [10] models are widely used. Recently, Lee et al. [26] adopted the Faster RCNN model in [1] to obtain regional visual features.

Attention mechanism provides a way to focus on certain aspects of the data in accordance with task-specific context. Several works $[14,19,26,30]$ exploited different attention mechanisms for aligning visual objects and textual words. These attention mechanisms can be categorized by the type of \{Query, Key, Value $\}$ [36]. With intra-modal attention, all of them are under the same modality. For example, in [30], the textual and visual content are encoded through two intra-modal memory networks separately for matching image and text. Whereas in [14], Huang et al. proposed a contextmodulated inter-modal attention mechanism to attend to pairwise instances of image and sentence. In [26], Lee et al. presented an inter-modal attention mechanism to associate RNN-based textual features and regional visual features.

Most recent works leverage ranking losses [6, 7, 18, 22, 23, 37, 39, 40] for learning visual-semantic embedding spaces. Frome et al. [7] associated cross-modal representations with a structured objective which encourages the distance between the matched image-text pair was smaller than that between the mismatched pair. Vendrov et al. [37] proposed an objective which could preserve the partial order structure of a visual-semantic hierarchy. In [22], Kiros et al. proposed to use a triplet ranking loss to penalize the model by averaging the individual violations across the negatives. This work set the tone for using triplet ranking loss, which has become a common ranking objective to learn joint embeddings for cross-modal retrieval. In [39, 40], Wang et al. extended the cross-view ranking loss with the within-view neighborhood structure-preserving constraints to preserve the structure in the joint embedding space. In VSE++ [6], Faghri et al. modified the pair-wise ranking loss based on violations caused by the hard negative examples (i.e., the closest non-matching query to the training query). This approach has been empirically shown to achieve significant improvement.

We approach the problem of joint embedding space learning from a different perspective. We investigate which type of information is important and has been captured or missed by current crossmodal retrieval models. Motivated by the insights, we then design our object-oriented inter- and intra-modal attention networks to address the characteristics of visual-semantic embeddings.

\section{WHAT DO THE MODELS LEARN FOR CROSS-MODAL RETRIEVAL?}

To shed light into the black boxes of cross-modal retrieval models and improve them, we present analyses through adversarial probing inspired by $[4,16]$. Different from these works, we jointly leverage adversarial inputs at the training phase and the leave-one-out technique for cross-validation to probe and quantify the importance of different types of information. Essentially, we are interested in how the state-of-the-art models utilize textual information to correlate the visual and textual context. In practice, we conduct experiments where various aspects of the input sentences are perturbed for training and measure the performance drop in comparison to the original model to quantify the importance of syntactic structure and lexical information.

\subsection{Methodology}

In the perturbation experiments, we take the publicly available codes and the features from the two representative state-of-the-art models: SCAN [26] and VSE++ [6]. We train them from scratch with the best settings available. Both of the models utilize RNN as the text encoders in a hope that dependencies among the word tokens in a sentence could be captured and encoded accordingly. SCAN performs fine-grained alignments between encoded word tokens and visual objects. In contrast, VSE++ utilizes the last hidden state of RNN as the sentence embeddings. For visual embeddings, SCAN uses pre-extracted regional visual features from a Faster RCNN in [1] while VSE++ fine-tunes a ResNet [10].

We examine the models with the standard training, validation, and testing splits in Flickr30K [42]. The models are evaluated using 
Table 1: Performance comparison of the models trained with perturbed inputs in the text-to-image retrieval task on Flickr30K. To understand what do the models learn, in each experiment, one type of perturbation is applied to the training textual inputs. Validation and testing inputs are without perturbation.

\begin{tabular}{llll|lll}
\hline & & SCAN [26] & & & VSE++ [6] \\
& $\mathrm{R} @ 1(\Delta, \Delta \%)$ & $\mathrm{R} @ 5(\Delta, \Delta \%)$ & $\mathrm{R} @ 10(\Delta, \Delta \%)$ & $\mathrm{R} @ 1(\Delta, \Delta \%)$ & $\mathrm{R} @ 5(\Delta, \Delta \%)$ & $\mathrm{R} @ 10(\Delta, \Delta \%)$ \\
\hline \hline Original model & $44.6(0.0,-0.0 \%)$ & $74.1(0.0,-0.0 \%)$ & $82.5(0.0,-0.0 \%)$ & $39.3(0.0,-0.0 \%)$ & $68.8(0.0,-0.0 \%)$ & $79.0(0.0,-0.0 \%)$ \\
\hline Random shuffling & $42.5(-2.1,-4.7 \%)$ & $71.2(-2.9,-3.9 \%)$ & $81.3(-1.2,-1.5 \%)$ & $37.3(-2.0,-5.0 \%)$ & $65.6(-3.2,-4.6 \%)$ & $76.5(-2.5,-3.2 \%)$ \\
Drop Numbers & $43.9(-0.7,-1.6 \%)$ & $73.5(-1.0,-1.3 \%)$ & $81.7(-0.8,-1.0 \%)$ & $38.5(-0.8,-2.1 \%)$ & $67.4(-1.4,-2.0 \%)$ & $77.5(-1.5,-1.9 \%)$ \\
Drop Prepositions & $42.2(-2.3,-5.2 \%)$ & $71.0(-3.1,-4.2 \%)$ & $80.0(-2.5,-3.0 \%)$ & $37.5(-1.8,-4.6 \%)$ & $67.0(-1.8,-2.6 \%)$ & $76.8(-2.2,-2.8 \%)$ \\
Drop Verbs & $41.3(-3.3,-7.4 \%)$ & $70.3(-3.8,-5.1 \%)$ & $79.5(-3.0,-3.6 \%)$ & $36.2(-3.1,-7.9 \%)$ & $65.5(-3.3,-4.8 \%)$ & $76.2(-2.6,-3.3 \%)$ \\
Drop Adjectives & $38.6(-6.0,-13.5 \%)$ & $67.5(-6.6,-8.9 \%)$ & $75.8(-6.7,-8.1 \%)$ & $34.0(-5.3,-13.5 \%)$ & $63.3(-5.5,-8.0 \%)$ & $72.4(-6.6,-8.4 \%)$ \\
Drop Nouns & $9.8(-34.8,-78 \%)$ & $28.5(-45.6,-62 \%)$ & $37.1(-45.4,-55 \%)$ & $9.5(-29.8,-76 \%)$ & $25.7(-43.1,-63 \%)$ & $34.5(-44.5,-56 \%)$ \\
\hline
\end{tabular}

Recall at $k(\mathrm{R} @ k)$ metric in the text-to-image retrieval task. In the training phase, we deliberately block the model from learning a specific type of information in the data. In each run, we apply one type of perturbation to all the training sentences. The perturbation is either random shuffling of word order or dropping one type of part-of-speech (POS) tagging in the sentences. For example, we trained the model with the sentence "shorts in dressed a baby blue ..." which is randomly shuffled from "a baby dressed in blue shorts ..." In the validation and testing phase, the models are evaluated with original non-perturbed sentences. As a consequence, the model trained with perturbed inputs will perform worse than the original model. The performance degradation then provides informative clues for the importance of the type of information dropped. A large drop in performance indicates that the blocked information is critical whereas a small drop implies irrelevance. More details about the experimental setup can be found in $\S 5.2$.

\subsection{Importance of Syntactic Structure}

Word order and syntactic structure in English is important for human to correctly understand the meaning of a sentence. Previous studies have demonstrated that encoders with recurrent architectures can capture syntactic structures and perform well in multiple text-only tasks such as named entity recognition [25], sentiment analysis [43], and machine translation [2]. In this study, we investigate whether encoding syntactic structures contributes to learning better visual-semantic embeddings for cross-modal retrieval.

Table 1 summarizes the impacts of perturbation for the two models of concern. Comparing the performance drop $(\Delta)$ and its relative percentage $(\Delta \%)$ between the baseline and the model trained with randomly shuffled inputs, the drops in all metrics are surprisingly little ( $\leq 5 \%$ for R@1) for both of the models. The results show that purging the syntactic information does not bring severe degradation. In other words, even with RNNs, these models only minimally utilize syntactic information in learning the joint embedding space for aligning sentences and images.

\subsection{Importance of Lexical Information}

What do cross-modal retrieval models learn if syntactic structure is unimportant for learning the joint embedding space? We suspect that the insignificance of syntax may also imply that spatial relationships (e.g., on, in, at) which are usually described by prepositional words $(I N)$ in the sentences may also be irrelevant. For verification, we conduct the leave-one-POS-out experiments to quantify the importance of different types of lexical information. The prior probabilities for a lexical type to appear in a sentence are: Cardinal Numbers:21.3\%, Prepositions: 92.8\%, Verbs: 91.5\%, Adjectives/Adverbs: $86.6 \%$, Nouns: $99.9 \%$ in Flickr30K.

From Table 1 we observe huge differences among the performance drops between the original model and the model trained with inputs discarding specific type of POS tagging. Although the prior probability is low, cardinal numbers $(C D)$ may not be learnt by the models. Unsurprisingly, even with a higher prior probability, prepositions $(I N)$ and spatial relationships in the sentences are ignored by the model. Notably, the verbal information is also not useful. The models do not fully capture the described action.

In contrast, we observe that adjectives and nouns plays important roles in cross-modal retrieval. Even with a relatively smaller prior, dropping adjectives results in more than $13 \%$ performance drop in R@1 for both of the models. As expected, the most important lexical type for cross-modal retrieval is noun.

\subsection{Insights from the Perturbation Experiments}

On the basis of the significance of adjectives and nouns, we conclude that those models mainly learn to leverage noun phrases to bridge across modalities in the joint embedding space. A bridge connects two shores. At the textual side, noun phrase are the proper units for encoding and linking. At the visual side, as simple descriptors (e.g., red) and visible objects (e.g., apple) are most often described by adjectives and nouns, visual objects are likely to be the proper units. The superiority of SCAN [26] over [6] may result from the regional object-level visual features extracted by Faster RCNN [34]. Driven by this observation, we argue that the alignments in the joint embedding space are likely to be object-oriented. We expect a model promoting object-oriented links would help to learn a better joint embedding space for cross-modal retrieval.

\section{THE PROPOSED MODEL}

As demonstrated in the perturbation experiments, utilizing objectoriented textual and visual context is evidently crucial for crossmodal retrieval. Following this insight, we propose to jointly use $\mathrm{CNN}$ for encoding textual context and Faster RCNN for visual 


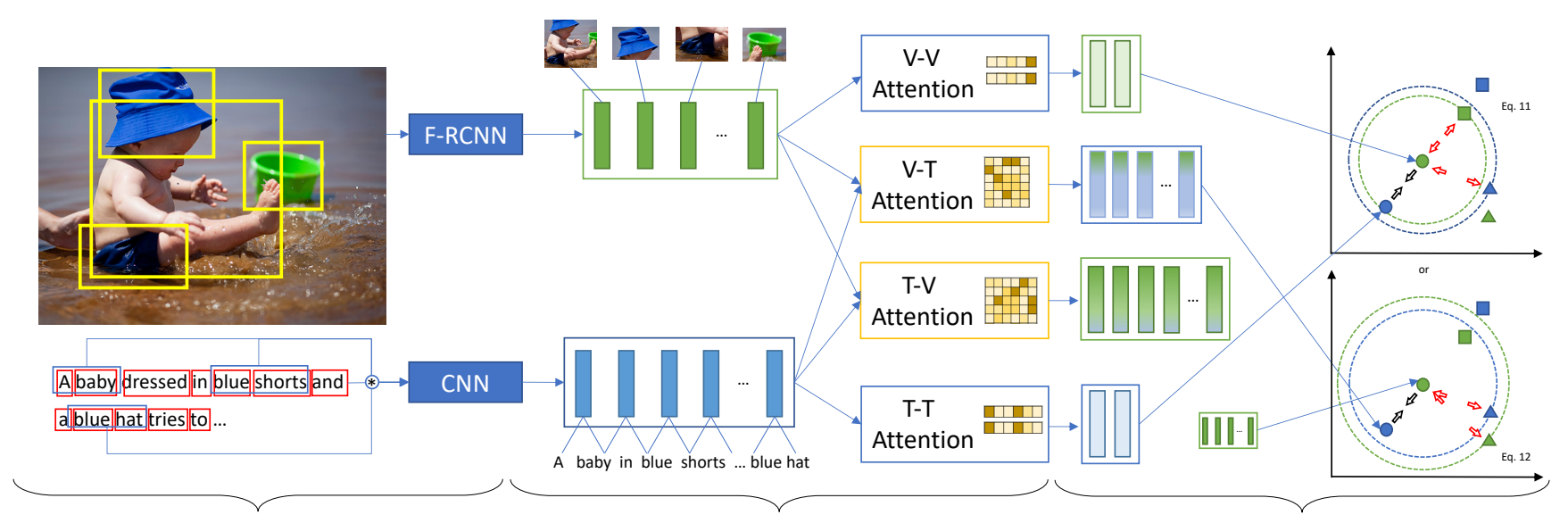

(a) Object-oriented encoders

(b) Inter- \& intra-modal attention networks

(c) Emphasis on inter- \& intra-modal hard negatives

Figure 1: The proposed object-oriented attention network (OAN) is composed of 3 components: (a) Object-oriented encoders (§ 4.1). (b) Inter- and intra-modal attention networks (\$ 4.2). (c) Inter- and intra-modal hard negative mining (\$ 4.3). Convolution kernels for uni-gram and bi-gram are colored in red and blue. Visual and textual embeddings are colored in green and blue, respectively. Attention weights are proportional to the darkness in yellow. Different shapes in (c) indicate different instances. (Better viewed in color.)

context. We propose to use inter-modal attention to refine, compare, and align these regionally-encoded objects. On the other hand, global context, which encodes interactions among multiple regional objects is not well-captured by RNN in [6] or CNN with max pooling in $[28,44]$. To address this issue, we propose to employ a multi-head context-aware intra-modal attention network to distill important global context for alignment. We show that incorporating both regional and global context with inter- and intra-modal attention will learn better visual-semantic embeddings. Additionally, we propose a new triplet objective which emphasizes inter and intra-modal harder negatives to retain both the cross-view and the within-view structure in the joint embedding space. We term the proposed model object-oriented attention network (OAN). The overall architecture is illustrated in Fig. 1.

\subsection{Object-Oriented Encoders}

For representing textual objects (i.e., noun phrases), previous works used RNNs [6, 14, 17, 18, 26, 30] or recursive neural network [31] traversing the parsing tree to encode word sequences. However, we empirically find that simple CNN-based encoders will better capture phrases and achieve superior performance. Given a sentence $\mathcal{S}=$ $\left[t_{1} \ldots t_{N}\right]$, we encode the word through an embedding matrix $x_{i}=$ $\mathbf{W}_{W} t_{i}, i \in\{1 \ldots N\}$. Then for each time step, we feed the word embeddings in the sentence to a set of 1-D convolution kernels $\left\{\mathbf{K}_{j}\right\}, j \in\{1,2,3,5,7\}$. Formally, we have:

$$
\mathbf{H}^{j}=\tanh \left(\mathbf{K}_{j} * \mathbf{X}+b_{j}\right)
$$

where "*” is the convolution operation and $b_{j}$ is the bias. The $j$-th convolutional kernels aim to capture features corresponding to uni-grams $(j=1)$, bi-grams $(j=2)$ and so on. In each time step $i$, we concatenate the outputs $h_{i}^{j}$ into $h_{i}=h_{i}^{1}\left\|h_{i}^{2}\right\| h_{i}^{3}\left\|h_{i}^{5}\right\| h_{i}^{7}$ and transform it to the textual embedding $e_{i} \in \mathbb{R}^{D}$ via a single-layer perceptron parameterized by $\mathbf{W}_{T}$ and $b_{T}$. The $i$-th textual object embedding $e_{i}$ is:

$$
e_{i}=\tanh \left(\mathbf{W}_{T} h_{i}+b_{T}\right)
$$

For representing visual objects, we follow the practice in [1] to localize salient objects with Faster RCNN [34] and extract regional features with a ResNet-101 [10] backbone. For an image $\mathcal{U}$, we use the pre-trained model provided by [1] to detect $M$ visual objects with top confidence scores and get the regional features for each object. With $\mathcal{U}=\left\{u_{1} \ldots u_{M}\right\}, u_{j} \in \mathcal{R}^{D_{V}}$ from Faster RCNN, we then apply a single-layer perceptron parameterized by $\mathbf{W}_{V}$ and $b_{V}$ to map them into $D$-dimensional embeddings. The $j$-th visual object embedding $v_{j}$ is:

$$
v_{j}=\tanh \left(\mathbf{W}_{V} u_{j}+b_{V}\right)
$$

\subsection{Inter-Modal and Intra-Modal Attention}

The inter-modal attention is effective to build regional connections between textual and visual objects. However, those inter-modal connections are not equally important and the interactions between objects within the same modality are missing. Intra-modal attention, as firstly introduced by [30] in the context of cross-modal retrieval with a multi-hop memory network, serves as a pooling function to pack the regional context with a global view. We propose to jointly leverage these two types of attention to better aligning objects in the two modalities.

Inter-modal attention: There are two directions in inter-modal attention: textual-to-visual (T-V) and visual-to-textual (V-T) attention. The goal of applying inter-modal attention is to transform the embedding of an object in one modality according to its relevance to the objects from the other modality. By minimizing the distance between the attended embedding and the original embedding, the model achieves fine-grained alignments between objects in different modalities in the joint embedding space. The attention networks take the outputs from the object-oriented encoders: 
$\mathcal{E}=\left\{e_{1}, \ldots, e_{N}\right\}, e_{i} \in \mathbb{R}^{D}$ and $\mathcal{V}=\left\{v_{1}, \ldots, v_{M}\right\}, v_{j} \in \mathbb{R}^{D}$. Let $s_{i j}=\frac{\left[e_{i}^{T} v_{j}\right]_{+}}{\left\|e_{i}\right\|\left\|v_{j}\right\|}$ denotes the similarity between the $i$-th textual phrase and the $j$-th visual object, where $i \in\{1, \ldots, N\}, j \in\{1, \ldots, M\}$ and $[.]_{+}=\max (., 0)$ is the hinge function.

In V-T attention, visually-attended textual embeddings $e^{V}$ are generated as a weighted combination of textual embeddings. The visually-attended weights are calculated by the softmax function with a temperature parameter $\lambda$. Specifically,

$$
\begin{aligned}
& w_{i j}=\operatorname{softmax}\left(\lambda \hat{s}_{i j}\right), \\
& e_{j}^{V}=\sum_{i=1}^{N} w_{i j} e_{i}, j \in\{1 \ldots M\},
\end{aligned}
$$

where $\hat{s}_{i j}=s_{i j} /\left\|s_{i},\right\|_{2}$. Similarly, for T-V attention, we generate the textually-attended visual embeddings $v_{i}^{T}$ as a weighted combination of visual embeddings with $\bar{s}_{i j}=s_{i j} /\left\|s_{i, j}\right\|_{2}$.

There are two sets of object-wise embeddings in each direction of inter-modal attention to represent an instance. For the V-T attention network, the instance-wise visual and textual embeddings are $v_{V T}=\left\{v_{1}, \ldots, v_{M}\right\}$ and $e_{V T}=\left\{e_{1}^{V}, \ldots, e_{M}^{V}\right\}$. For the T-V attention network, the instance-wise visual and textual embeddings are $v_{T V}=\left\{v_{1}^{T}, \ldots, v_{N}^{T}\right\}$ and $e_{T V}=\left\{e_{1}, \ldots, e_{N}\right\}$, respectively.

Intra-modal attention: The intra-modal attention network focuses on certain aspects of data with respect to intra-modal context. In contrast to [30], we leverage a single-hop multi-head contextaware attention network to capture the interactions between objects with a global view and to distill informative objects for alignment from individual modalities. Let $c_{T}=\frac{1}{N} \sum_{i} e_{i}$ and $c_{V}=\frac{1}{M} \sum_{j} v_{j}$ denote the the textual and visual context respectively. For $k$-th head of textual-to-textual attention (T-T), we define the $k$-th textually attended textual embedding as a weighted combination of textual embeddings:

$$
\begin{aligned}
h_{i} & =\tanh \left(\mathbf{W}_{c t}^{k} c_{T}\right)^{T} \tanh \left(\mathbf{W}_{t}^{k} e_{i}\right), \\
w_{i}^{k} & =\operatorname{softmax}\left(\lambda h_{i}\right), \\
e_{T T}^{k} & =\sum_{i=1}^{N} w_{i}^{k} e_{i}
\end{aligned}
$$

Similarly, we generate $k$-th visually attended visual embedding $v_{V V}^{k}$ with visual-to-visual attention $(\mathrm{V}-\mathrm{V})$. The final instance-wise intra-modal attended representations are: $e_{T T}=\left\{e_{T T}^{1}, \ldots, e_{T T}^{K}\right\}$ and $v_{V V}=\left\{v_{V V}^{1}, \ldots, v_{V V}^{K}\right\}$ for the textual and visual part, respectively.

\subsection{Emphasis on Inter-Modal and Intra-Modal Hard Negatives}

For learning a visual-semantic embedding space, most prior works including $[18,22,40]$ minimize a hinge-based triplet loss to encourage a similarity margin $\alpha$ between the paired visual-textual embeddings and the non-paired ones. Instead of taking summation over all the non-paired instances in the triplet loss, it was empirically shown in [6] that taking only the hard negatives is more efficient and robust. Formally, let $(v, t)$ denotes a paired textual-visual embeddings and $v^{-}$and $t^{-}$denote the non-matching embeddings corresponding to $v, t$. With the hard negatives defined as $\hat{v}=\underset{v-}{\operatorname{argmax}} S\left(v^{-}, t\right)$ and $\hat{t}=\underset{t^{-}}{\operatorname{argmax}} S\left(v, t^{-}\right)$, the triplet loss emphasizing inter-modal hard negative samples can be written as:

$$
l(v, t)=\sum_{v}[\alpha-S(v, t)+S(v, \hat{t})]_{+}+\sum_{t}[\alpha-S(v, t)+S(\hat{v}, t)]_{+},
$$

where $S(v, t)$ measures the distance between them, and $[.]_{+}=$ $\max (., 0)$ is the hinge function.

To further promote intra-modal discrepancies, we propose to incorporate both the inter-model hard negative examples and the intra-model hard negative examples. Essentially, given $t$, we sample the intra-modal hard negative example $\bar{t}$ paired to either 1) the original text embedding $t$ or 2) the text part of the hard visual embedding $\hat{v}$. These two types of intra-modal hard negative examples are illustrated in Fig. 1-(c). Let $\bar{t}$ and $\bar{v}$ denote the visual and textual intra-modal hard negative examples, we introduce two additional triplet losses $\alpha-S(v, v)-S(v, \bar{v})$ and $\alpha-S(t, t)-S(t, \bar{t})$ to Eq. 9 weighted by a hyper parameter $\beta$. Since $S(t, t)=S(v, v)=1$. We propose the following loss function:

$$
\begin{aligned}
l(v, t) & =\sum_{v}\left\{\left[\alpha_{\text {inter }}-S(v, t)+S(v, \hat{t})\right]_{+}+\beta\left[\alpha_{\text {intra }}-S(v, \bar{v})\right]_{+}\right\} \\
& +\sum_{t}\left\{\left[\alpha_{\text {inter }}-S(v, t)+S(\hat{v}, t)\right]_{+}+\beta\left[\alpha_{\text {intra }}-S(t, \bar{t})\right]_{+}\right\}
\end{aligned}
$$

As depicted in Fig. 1-(C), we sample $\bar{t}$ and $\bar{v}$ as either the hard negative to the original textual and visual embedding:

$$
\bar{v}=\underset{v^{-}}{\operatorname{argmax}} S\left(v^{-}, v\right), \bar{t}=\underset{t^{-}}{\operatorname{argmax}} S\left(t^{-}, t\right),
$$

or the corresponding visual part of the hard negative textual embeddings and the corresponding textual part of the hard negative visual embeddings:

$$
\begin{aligned}
\bar{v} & =v_{\hat{t}}, \hat{t}=\underset{t^{-}}{\operatorname{argmax}} S\left(v, t^{-}\right), \\
\bar{t} & =t_{\hat{v}}, \hat{v}=\underset{v^{-}}{\operatorname{argmax}} S\left(v^{-}, t\right)
\end{aligned}
$$

\subsection{Training and Inference}

Let the set-wise cosine similarity $S(a, b)=\frac{1}{|a|} \sum_{i=0}^{|a|} \frac{a_{i}^{T} b_{i}}{\left\|a_{i}\right\|\left\|b_{i}\right\|}$ as the distance measure. With the attended embedding sets $e_{T T}, v_{V V}$ from the intra-modal attention and $v_{T V}, e_{T V}, v_{V T}, e_{V T}$ from the inter-modal attention, the final optimization problem is:

$$
\min _{\theta}(1-\gamma)\left[l\left(v_{V T}, e_{V T}\right)+l\left(v_{T V}, e_{T V}\right)\right]+\gamma l\left(v_{V V}, e_{T T}\right),
$$

where $\theta$ is the trainable parameters in the model and $\gamma$ balances the importance of inter- and intra-attention networks. At inference time, for retrieving instances in another modality, after mapping the textual/visual query to the joint embedding space, the models rank the semantic closeness according to:

$$
r(v, t)=(1-\gamma)\left[S\left(v_{V T}, e_{V T}\right)+S\left(v_{T V}, e_{T V}\right)\right]+\gamma S\left(v_{V V}, e_{T T}\right) .
$$




\section{EXPERIMENTS}

This section evaluates the proposed object-oriented inter- and intramodal attention network (OAN) for cross-modal retrieval. Given a query sentence (resp. image), the goal is to find the corresponding image (resp. sentence) in the dataset.

\subsection{Datasets and Evaluation Metric}

Flickr30K [42]. There are 31,783 images in Flickr30K where five captions are available for each image. For experiments on Flickr30K, we use the same split defined in $[6,18,26]$ with 29,000 training, 1,000 validation, and 1,000 testing images.

MS-COCO [27]. The MS-COCO dataset contains 123,287 images where each image is annotated with five captions. For the splits we follow the commonly compared splits as defined in [6, 26, 38, 44] to move originally left 30,504 validation images to the training set, resulting a training set of 118,287 images. We report and compare either the average of metrics on five folds of 1,000 testing set or the metrics on the whole 5,000 testing set.

Metric. We adopt the standard evaluation criteria used in most prior work on cross-modal retrieval tasks [6, 26, 30, 44]. We measure rank-based performance by Recall at K (R@k). Given a query, R@k calculates the percentage of test instances for which the correct one can be found in the top- $K$ retrieved instances. We report $k=1,5,10$.

\subsection{Experimental Setup}

To identify and represent salient visual object in images, we use the code and the model provided by [1] to detect visual objects and extract their corresponding features. The visual feature is with shape $36 \times 2048$, where 36 is the number of region of interest in an image and 2048 is the dimension of the flattened 5-th pooling layer of the ResNet [10] backbone in Faster RCNN [34]. We use raw the features without 12 normalization.

We set the embedding dimension $D$ to 512 where the same dimension is shared by all the context vectors in the attention modules. We use 300-dim randomly initialized word embeddings. For text preprocessing, we tokenize, lower-case, truncate maximum sentence length to 60 on MS-COCO and 80 on Flickr30K, and then remove word tokens which appear less than 4 times. All the other weights within the network are initialized with the Xavier initialization [8] The softmax temperatures are $\lambda_{T T}=1.0, \lambda_{V V}=1.0, \lambda_{T V}=0.9$, $\lambda_{V T}=0.5$. Other hyper-parameters are set as follows: $\alpha_{\text {inter }}=0.2$, $\alpha_{\text {intra }}=0.1, \beta=0.5, K=6$, and $\gamma=0.3$.

For training, we use Adam [21] optimizer with a $5 \times 10^{-4}$ learning rate then $5 \times 10^{-5}$ after 15 -th epoch. Models with best R@1 in the validation set are selected for testing. Weight decay is set to $10^{-6}$ and gradients larger than 2.0 are clipped. The batch size is 128 . We train 24 epochs on all the datasets. We implemented our model using PyTorch. Our machine has a Intel E5-2660v3 CPU, 64 GB RAM, and one NVIDIA Titan Xp GPU. Training the model takes roughly 6 hours on Flickr30K and 24 hours on MS-COCO.

For the perturbation experiments in $\S 3$, we use the publicly available codes and features from $[6,26]$ and follow the suggested settings to reproduce the best results if possible. For all the baselines in the main results ( $§ 5.4)$ we report and compare the best single model which achieves the highest R@1 on the text-to-image retrieval task. For fair comparison, we do not consider the performance with model ensemble.
Table 2: Ablation studies of the proposed model for text-toimage retrieval in the $1 \mathrm{~K}$ testing set of Flickr30K.

\begin{tabular}{lccc}
\hline & R@1 & R@5 & R@10 \\
\hline \hline Full model (Eq. 10 and Eq. 11) & 53.1 & 79.9 & 86.8 \\
Full model (Eq. 10 and Eq. 12) & $\mathbf{5 3 . 3}$ & $\mathbf{8 0 . 1}$ & $\mathbf{8 7 . 1}$ \\
\hline No intra-modal hard negatives & 52.8 & 79.6 & 86.8 \\
No inter-modal attention & 48.2 & 77.5 & 85.5 \\
No intra-modal attention & 47.8 & 77.0 & 85.2 \\
No attention & 37.2 & 70.7 & 76.2 \\
No object-oriented visual encoder & 41.2 & 70.9 & 80.0 \\
No object-oriented textual encoder & 51.0 & 78.5 & 85.2 \\
\hline
\end{tabular}

\subsection{Ablation Studies}

We perform ablation studies in the text-to-image retrieval task on the Flickr30K testing set to quantify the contribution of each component, Each time we remove one component and measure its relative performance drop. The larger the degradation implies that it is more important. Table 2 summarizes the result. On the top two rows we list the full model with the two sampling strategies for intra-modal hard negative examples in the proposed objective. For the experiment without object-oriented textual encoder, we swap text CNN to Bi-directional LSTM. For the experiment without object-oriented visual encoder, we follow [30] to resize images to $448 \times 448$ and use ResNet-152 to extract $14 \times 14 \times 2048$ grid-based visual features. In the experiments without attention we apply mean pooling over the encoded objects in individual modalities directly.

Object-oriented encoders. Textual phrases and visual objects are center to visual-semantic embeddings. The $\mathrm{CNN}$ architecture in the textual encoder captures important linguistic priors such as n-gram structures and noun phrases. On the other hand, the object detection network (Faster RCNN), which can be analogized to the inherited natural attention in human's cognitive system, helps the model to focus on the salient visual objects. Empirically the visual encoder plays a more important role than textual encoder. Both of them are crucial to achieve the best performance.

Inter- and intra-modal attention mechanisms. Instead of bluntly aligning all the context in the joint embedding space, the attention mechanisms serve as the crucial approach to pool and connect encoded objects. The model without attention results in the worst performance in our ablation experiments. We note that models with inter-modal and intra-modal attention yield comparable performance. The two types of attention together contribute to the top-performing model in a complementary manner.

Intra-modal hard negative examples. Preservation of the intra-modal structure achieves consistent gain but it is not as significant as other components. The training objective considering the paired intra-modal content of the sampled inter-modal hard negative (Eq. 12) results in slightly higher performance than the objective considering the hard intra-modal content (Eq. 11).

\subsection{Quantitative Results}

We compare various research from classic models $[18,41]$ to recent models with advanced visual features $[6,14,39,40]$ and attention mechanisms $[9,15,26,30]$. For reference, we also list their corresponding textual and visual encoders. We either directly report the 
Table 3: Performance comparison on Flickr30K's 1K testing set. For each baseline, the best single model with highest R@1 in text-to-image retrieval task is reported and compared. We also list the backbone encoders (textual encoder, visual encoder).

\begin{tabular}{|c|c|c|c|c|c|c|}
\hline & \multicolumn{3}{|c|}{ Text-to-Image Retrieval } & \multicolumn{3}{|c|}{ Image-to-Text Retrieval } \\
\hline & R@1 & R@5 & R@10 & R@1 & R@5 & $\mathrm{R} @ 10$ \\
\hline DCCA [41] (TF-IDF, AlexNet) & 12.6 & 31.0 & 43.0 & 16.7 & 39.3 & 52.9 \\
\hline DVSA [18] (RNN, AlexNet) & 15.2 & 37.7 & 50.5 & 22.2 & 48.2 & 61.4 \\
\hline SM-LSTM [14] (RNN, VGG) & 30.2 & 60.4 & 72.3 & 42.5 & 71.9 & 81.5 \\
\hline 2WayNet [5] (GMM+HGLMM, VGG) & 36.0 & 55.6 & - & 49.8 & 67.5 & - \\
\hline $\mathrm{VSE}++[6](\mathrm{RNN}, \mathrm{ResNet})$ & 39.6 & - & 79.5 & 52.9 & - & 87.2 \\
\hline DPC [44] (CNN, ResNet) & 39.1 & 69.2 & 80.9 & 55.6 & 81.9 & 89.0 \\
\hline DAN [30] (RNN, ResNet) & 39.4 & 69.2 & 79.1 & 55.0 & 81.8 & 89.5 \\
\hline SCO [15] (RNN, ResNet) & 41.1 & 70.5 & 80.1 & 55.5 & 82.0 & 89.3 \\
\hline SCAN [26] (RNN, FRCNN-ResNet) & 45.8 & 74.4 & 83.0 & 61.8 & 87.5 & 93.7 \\
\hline OAN (Ours) (CNN, FRCNN-ResNet) & 53.3 & 80.1 & 87.1 & 68.6 & 93.0 & 96.0 \\
\hline
\end{tabular}

Table 4: Performance comparison on MS-COCO's $1 \mathrm{~K}$ and $5 \mathrm{~K}$ testing sets. For each baseline, the best single model with highest $\mathrm{R} @ 1$ in text-to-image retrieval task is reported and compared.

\begin{tabular}{|c|c|c|c|c|c|c|}
\hline & \multicolumn{3}{|c|}{ Text-to-Image Retrieval } & \multicolumn{3}{|c|}{ Image-to-Text Retrieval } \\
\hline & R@1 & R@5 & $\mathrm{R} @ 10$ & $\mathrm{R} @ 1$ & R@5 & R@10 \\
\hline & \multicolumn{6}{|c|}{$1 \mathrm{k}$ Testing Images } \\
\hline DVSA [18] (RNN, AlexNet) & 27.4 & 60.2 & 74.8 & 38.4 & 69.9 & 80.5 \\
\hline SM-LSTM [14] (RNN, VGG) & 30.2 & 60.4 & 72.3 & 42.5 & 71.9 & 81.5 \\
\hline Order-embeddings [38] (RNN, VGG) & 37.9 & - & 85.9 & 46.7 & - & 88.9 \\
\hline 2WayNet [5] (GMM+HGLMM, VGG) & 39.7 & 63.3 & - & 55.8 & 75.2 & - \\
\hline $\mathrm{VSE}++[6]$ (RNN, ResNet) & 52.0 & - & 92.0 & 64.6 & - & 95.7 \\
\hline DPC [44] (CNN, ResNet) & 47.1 & 79.9 & 90.0 & 65.6 & 89.8 & 95.5 \\
\hline GXN [9] (RNN, ResNet) & 56.6 & - & 94.5 & 68.5 & - & 97.9 \\
\hline SCO [15] (RNN, ResNet) & 56.7 & 87.5 & 94.8 & 69.9 & 92.9 & 97.5 \\
\hline SCAN [26] (RNN, FRCNN-ResNet) & 56.4 & 87.0 & 93.9 & 70.9 & 94.5 & 97.8 \\
\hline \multirow[t]{2}{*}{ OAN (Ours) (CNN, FRCNN-ResNet) } & 60.2 & 88.6 & 94.5 & 71.7 & 96.4 & 99.3 \\
\hline & \multicolumn{6}{|c|}{ 5k Testing Images } \\
\hline DVSA [18] (RNN, AlexNet) & 10.7 & 29.6 & 42.2 & 16.5 & 39.2 & 52.0 \\
\hline Order-embeddings [38] (RNN, VGG) & 31.7 & - & 74.6 & 23.3 & - & 84.7 \\
\hline $\mathrm{VSE}++[6](\mathrm{RNN}, \mathrm{ResNet})$ & 30.3 & - & 72.4 & 41.3 & - & 81.2 \\
\hline DPC [44] (CNN, ResNet) & 25.3 & 53.4 & 66.4 & 41.2 & 70.5 & 81.1 \\
\hline GXN [9] (RNN, ResNet) & 31.7 & - & 74.6 & 42.0 & - & 84.7 \\
\hline SCO [15] (RNN, ResNet) & 33.1 & 62.9 & 75.5 & 42.8 & 72.3 & 83.0 \\
\hline SCAN (RNN, FRCNN-ResNet) & 34.4 & 63.7 & 75.7 & 46.4 & 77.4 & 87.2 \\
\hline OAN (Ours) (CNN, FRCNN-ResNet) & 37.0 & 66.6 & 78.0 & 47.8 & 81.2 & 90.4 \\
\hline
\end{tabular}

scores of the best single model in the original paper or refer to the summarization made in [44]. Note that ensembled models reported in original papers are not compared for fairness.

Our full model includes object-oriented encoders and inter- and intra-modal attention networks. As analyzed with the ablation studies, we choose the full model trained with the loss function emphasizing paired intra-modal hard negative examples (Eq. 10 and Eq. 12). Table 3 shows the quantitative results on Flickr30K.

Since most models employ RNN as the textual encoder, the trend shows that models with more advanced visual features generally perform better. Interestingly, there are clear performance gaps when swapping the visual backbone from AlexNet [24] to VGG [35] or ResNet [10]. Sharing a similar object-oriented visual encoder (Faster RCNN) as SCAN [26], our model achieve superior performance to other models. Note that even with only ResNet backbone, our model still achieves comparable or superior performance to baselines with ResNet backbones as shown in Table 2 . With the object-oriented textual and visual encoders associated with interand intra-modal attention networks, our model achieves new stateof-the-art results on Flickr30K. OAN outperforms the previous best 


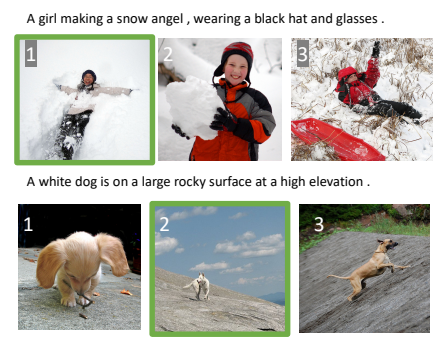

(a) Flickr30K: Text-to-image retrieval
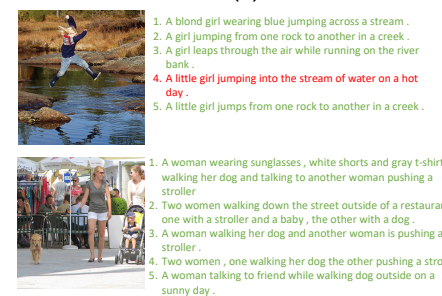

(c) Flickr30K: Image-to-text retrieval
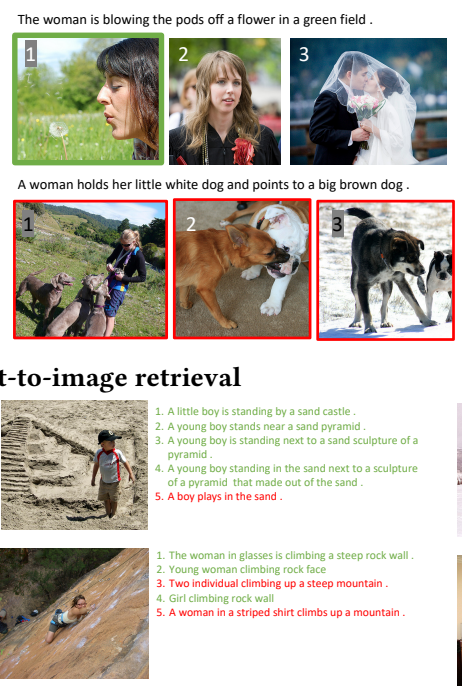
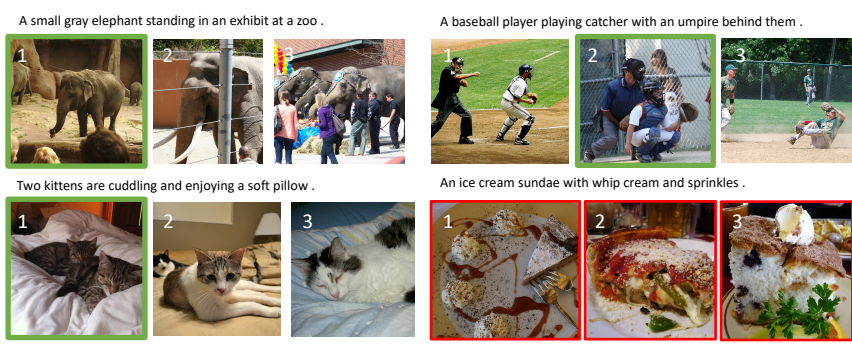

(b) MS-COCO: Text-to-image retrieval
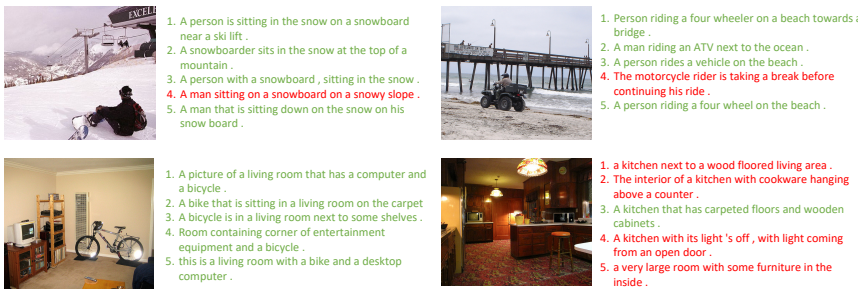

(d) MS-COCO: Image-to-text retrieval

Figure 2: Qualitative examples of the proposed model in cross-modal retrieval tasks on Flickr30K and MS-COCO. One failure case is shown at the bottom right corner of each task. The correct matches are colored in green.

model (SCAN [26]) by $7.5(16.3 \%), 5.7(7.6 \%), 4.1(4.9 \%)$ and $6.8(11 \%)$, 5.5 (6.2\%), 2.3 (2.4\%) in R@1, R@5 and R@10 for the text-to-image and the image-to-text retrieval tasks, respectively.

MS-COCO is roughly four times larger than Flickr30K. Following the protocol in [18], we report the evaluation results on the $1 \mathrm{~K}$ testing images (5-fold) and $5 \mathrm{~K}$ testing images. The quantitative results of the two splits on MS-COCO is shown in Table 4. On both testing splits, our model delivers the best cross-modal retrieval performance on most of the metrics.

\subsection{Qualitative Results}

Figure 2 illustrates some qualitative results from the image-to-text and text-to-image retrieval tasks on Flickr30K and MS-COCO. We provide four examples for each task and include one failure case at the bottom right corner. The top two rows show the top three retrieved images with the natural language query given above. The one and only one correct image is marked in green. The image-totext retrieval results are depicted in the bottom two rows. We list the top five retrieved sentences and the query image. The correct sentences are colored in green.

As can be observed, the images in Flickr30K and MS-COCO typically contain salient objects which are also described in the associating sentences. Our model successfully delivers high quality retrieval outputs in most cases. However, in the failure cases of text-to-image retrieval, we observe that the model either fails to associate some complex textual phrases or misses some multiconstraints to be satisfied. For the image-to-text retrieval, we note that the subtle difference between sentences and some important linguistic information (such as counting) are harder to be captured by the current model. On the other hand. we also observe that some wrongly retrieved sentences are still semantically consistent with the query image. This issue may be the inherited limitation as a consequence of the dataset bias.
Quantitatively and qualitatively, we demonstrate the superiority and the generalizability of the proposed model on multiple datasets. Extending our model to capture out-of-vocabulary inter-modal dependencies and tackle multi-constraint textual queries would be an important subject for future work.

\section{CONCLUSION}

We have presented analyses via perturbation experiments with random shuffling and leave-one-POS-out to investigate and quantify what cross-modal retrieval models learn. The results show that the regional alignments between textual phrases and visual objects play an important role in image-text matching while linguistic information such as syntactic structure and some lexical types was not well-captured even with RNN encoders. On the basis of this insight, we have proposed a new cross-modal retrieval model (OAN) which employs object-oriented encoders along with interand intra-modal attention networks to pool and align visual objects and text phrases both regionally and globally. A new inter- and intramodal structure preserving loss function has also been introduced to further enhance the intra-modal discrepancies. We have validated the effectiveness of the proposed model on the Flickr30K and MSCOCO datasets with new state-of-the-art results.

\section{ACKNOWLEDGEMENT}

This research is supported in part by DARPA grant FA8750-18-20018 funded under the AIDA program. This work is also supported in part through the financial assistance award 60NANB17D156 from U.S. Department of Commerce, National Institute of Standards and Technology and by the Intelligence Advanced Research Projects Activity (IARPA) via Department of Interior/Interior Business Center (DOI/IBC) contract number D17PC00340. The authors would like to thank the anonymous reviewers for their suggestions. 


\section{REFERENCES}

[1] Peter Anderson, Xiaodong He, Chris Buehler, Damien Teney, Mark Johnson, Stephen Gould, and Lei Zhang. 2018. Bottom-Up and Top-Down Attention for Image Captioning and Visual Question Answering. In CVPR.

[2] Dzmitry Bahdanau, Kyunghyun Cho, and Yoshua Bengio. 2014. Neural machine translation by jointly learning to align and translate. arXiv preprint arXiv:1409.0473 (2014).

[3] Kyunghyun Cho, Bart van Merrienboer, Caglar Gulcehre, Dzmitry Bahdanau, Fethi Bougares, Holger Schwenk, and Yoshua Bengio. 2014. Learning Phrase Representations using RNN Encoder-Decoder for Statistical Machine Translation. In Proceedings of the 2014 Conference on Empirical Methods in Natural Language Processing (EMNLP). 1724-1734.

[4] Volkan Cirik, Louis-Philippe Morency, and Taylor Berg-Kirkpatrick. 2018. Visual Referring Expression Recognition: What Do Systems Actually Learn?. In Proceedings of the 2018 Conference of the North American Chapter of the Association for Computational Linguistics: Human Language Technologies, Volume 2 (Short Papers). Association for Computational Linguistics, 781-787. http: //aclweb.org/anthology/N18-2123

[5] Aviv Eisenschtat and Lior Wolf. 2017. Linking Image and Text with 2-Way Nets In Computer Vision and Pattern Recognition (CVPR), 2017 IEEE Conference on. IEEE, 1855-1865.

[6] Fartash Faghri, David J Fleet, Jamie Ryan Kiros, and Sanja Fidler. 2018. VSE++ Improving Visual-Semantic Embeddings with Hard Negatives. (2018). https //github.com/fartashf/vsepp

[7] Andrea Frome, Gregory S. Corrado, Jonathon Shlens, Samy Bengio, Jeffrey Dean, Marc'Aurelio Ranzato, and Tomas Mikolov. 2013. DeViSE: A Deep VisualSemantic Embedding Model. In Advances in Neural Information Processing Systems 26: 27th Annual Conference on Neural Information Processing Systems 2013. Proceedings of a meeting held December 5-8, 2013, Lake Tahoe, Nevada, United States. 2121-2129.

[8] Xavier Glorot and Yoshua Bengio. 2010. Understanding the difficulty of training deep feedforward neural networks. In Proceedings of the thirteenth international conference on artificial intelligence and statistics. 249-256.

[9] Jiuxiang Gu, Jianfei Cai, Shafiq R Joty, Li Niu, and Gang Wang. 2018. Look, imagine and match: Improving textual-visual cross-modal retrieval with generative models. In Proceedings of the IEEE Conference on Computer Vision and Pattern Recognition. 7181-7189.

[10] Kaiming He, Xiangyu Zhang, Shaoqing Ren, and Jian Sun. 2016. Deep residual learning for image recognition. In Proceedings of the IEEE conference on computer vision and pattern recognition. $770-778$.

[11] Sepp Hochreiter and Jürgen Schmidhuber. 1997. Long short-term memory. Neural computation 9, 8 (1997), 1735-1780.

[12] Micah Hodosh, Peter Young, and Julia Hockenmaier. 2013. Framing image description as a ranking task: Data, models and evaluation metrics. Fournal of Artificial Intelligence Research 47 (2013), 853-899.

[13] Po-Yao Huang, Junwei Liang, Jean-Baptiste Lamare, and Alexander G. Hauptmann. 2018. Multimodal Filtering of Social Media for Temporal Monitoring and Event Analysis. In Proceedings of the 2018 ACM on International Conference on Multimedia Retrieval (ICMR '18). ACM, New York, NY, USA, 450-457. https://doi.org/10.1145/3206025.3206079

[14] Yan Huang, Wei Wang, and Liang Wang. 2017. Instance-Aware Image and Sentence Matching with Selective Multimodal LSTM. In 2017 IEEE Conference on Computer Vision and Pattern Recognition (CVPR). IEEE, 7254-7262.

[15] Yan Huang, Qi Wu, and Liang Wang. 2017. Learning semantic concepts and order for image and sentence matching. arXiv preprint arXiv:1712.02036 (2017).

[16] Robin Jia and Percy Liang. 2017. Adversarial Examples for Evaluating Reading Comprehension Systems. In Proceedings of the 2017 Conference on Empirical Methods in Natural Language Processing. 2021-2031.

[17] Xinyang Jiang, Fei Wu, Xi Li, Zhou Zhao, Weiming Lu, Siliang Tang, and Yueting Zhuang. 2015. Deep compositional cross-modal learning to rank via local-global alignment. In Proceedings of the 23rd ACM international conference on Multimedia. ACM, 69-78.

[18] Andrej Karpathy and Li Fei-Fei. 2015. Deep visual-semantic alignments for generating image descriptions. In Proceedings of the IEEE conference on computer vision and pattern recognition. 3128-3137.

[19] Andrej Karpathy, Armand Joulin, and Li F Fei-Fei. 2014. Deep fragment embeddings for bidirectional image sentence mapping. In Advances in neural information processing systems. 1889-1897.

[20] Yoon Kim. 2014. Convolutional Neural Networks for Sentence Classification. In Proceedings of the 2014 Conference on Empirical Methods in Natural Language Processing (EMNLP). 1746-1751.

[21] Diederik P Kingma and Jimmy Ba. 2014. Adam: A method for stochastic optimization. arXiv preprint arXiv:1412.6980 (2014).

[22] Ryan Kiros, Ruslan Salakhutdinov, and Richard S. Zemel. 2014. Unifying VisualSemantic Embeddings with Multimodal Neural Language Models. NIPS Workshop (2014).
[23] Benjamin Klein, Guy Lev, Gil Sadeh, and Lior Wolf. 2015. Associating neural word embeddings with deep image representations using Fisher Vectors. In IEEE Conference on Computer Vision and Pattern Recognition, CVPR 2015, Boston, MA, USA, fune 7-12, 2015. 4437-4446.

[24] Alex Krizhevsky, Ilya Sutskever, and Geoffrey E Hinton. 2012. Imagenet classification with deep convolutional neural networks. In Advances in neural information processing systems. 1097-1105.

[25] Guillaume Lample, Miguel Ballesteros, Sandeep Subramanian, Kazuya Kawakami, and Chris Dyer. 2016. Neural Architectures for Named Entity Recognition. In Proceedings of NAACL-HLT. 260-270.

[26] Kuang-Huei Lee, Xi Chen, Gang Hua, Houdong Hu, and Xiaodong He. 2018. Stacked Cross Attention for Image-Text Matching. arXiv preprint arXiv:1803.08024 (2018).

[27] Tsung-Yi Lin, Michael Maire, Serge Belongie, James Hays, Pietro Perona, Deva Ramanan, Piotr Dollár, and C Lawrence Zitnick. 2014. Microsoft coco: Common objects in context. In European conference on computer vision. Springer, 740-755.

[28] Lin Ma, Zhengdong Lu, Lifeng Shang, and Hang Li. 2015. Multimodal convolutional neural networks for matching image and sentence. In Proceedings of the IEEE international conference on computer vision. 2623-2631.

[29] Tomas Mikolov, Ilya Sutskever, Kai Chen, Greg S Corrado, and Jeff Dean. 2013. Distributed representations of words and phrases and their compositionality. In Advances in neural information processing systems. 3111-3119.

[30] Hyeonseob Nam, Jung-Woo Ha, and Jeonghee Kim. 2017. Dual Attention Networks for Multimodal Reasoning and Matching. In Computer Vision and Pattern Recognition (CVPR), 2017 IEEE Conference on. IEEE, 2156-2164.

[31] Zhenxing Niu, Mo Zhou, Le Wang, Xinbo Gao, and Gang Hua. 2017. Hierarchical multimodal lstm for dense visual-semantic embedding. In Computer Vision (ICCV), 2017 IEEE International Conference on. IEEE, 1899-1907.

[32] Jeffrey Pennington, Richard Socher, and Christopher Manning. 2014. Glove: Global vectors for word representation. In Proceedings of the 2014 conference on empirical methods in natural language processing (EMNLP). 1532-1543.

[33] Florent Perronnin and Christopher Dance. 2007. Fisher kernels on visual vocabularies for image categorization. In 2007 IEEE conference on computer vision and pattern recognition. IEEE, 1-8.

[34] Shaoqing Ren, Kaiming He, Ross Girshick, and Jian Sun. 2015. Faster r-cnn: Towards real-time object detection with region proposal networks. In Advances in neural information processing systems. 91-99.

[35] Karen Simonyan and Andrew Zisserman. 2014. Very deep convolutional networks for large-scale image recognition. arXiv preprint arXiv:1409.1556 (2014).

[36] Ashish Vaswani, Noam Shazeer, Niki Parmar, Jakob Uszkoreit, Llion Jones, Aidan N Gomez, Łukasz Kaiser, and Illia Polosukhin. 2017. Attention is all you need. In Advances in Neural Information Processing Systems. 5998-6008.

[37] Ivan Vendrov, Ryan Kiros, Sanja Fidler, and Raquel Urtasun. 2015. OrderEmbeddings of Images and Language. CoRR abs/1511.06361 (2015).

[38] Ivan Vendrov, Ryan Kiros, Sanja Fidler, and Raquel Urtasun. 2015. Orderembeddings of images and language. arXiv preprint arXiv:1511.06361 (2015).

[39] Liwei Wang, Yin Li, Jing Huang, and Svetlana Lazebnik. 2018. Learning twobranch neural networks for image-text matching tasks. IEEE Transactions on Pattern Analysis and Machine Intelligence (2018).

[40] Liwei Wang, Yin Li, and Svetlana Lazebnik. 2016. Learning Deep StructurePreserving Image-Text Embeddings. In Computer Vision and Pattern Recognition (CVPR), 2016 IEEE Conference on. IEEE, 5005-5013.

[41] Fei Yan and Krystian Mikolajczyk. 2015. Deep correlation for matching images and text. In Proceedings of the IEEE conference on computer vision and pattern recognition. 3441-3450

[42] Peter Young, Alice Lai, Micah Hodosh, and Julia Hockenmaier. 2014. From image descriptions to visual denotations: New similarity metrics for semantic inference over event descriptions. Transactions of the Association for Computational Linguistics 2 (2014), 67-78.

[43] Lei Zhang, Shuai Wang, and Bing Liu. 2018. Deep learning for sentiment analysis: A survey. Wiley Interdisciplinary Reviews: Data Mining and Knowledge Discovery (2018).

[44] Zhedong Zheng, Liang Zheng, Michael Garrett, Yi Yang, and Yi-Dong Shen. 2017. Dual-Path Convolutional Image-Text Embedding. CoRR abs/1711.05535 (2017). 